\title{
INTERVENCIÓN DE ENFERMERÍA EN EL AUTOCUIDADO CON APOYO EDUCATIVO EN PERSONAS CON DIABETES MELLITUS TIPO 2
}

Avila Alpirez, Hermelinda*; Meza Guevara, Susana**; Frías Reyna; Barbarita***; Sánchez Andrade; Ernestina***; Vega Alanís; Carmen*****; Hernández Saldivar, Ma Antonia******

*Profesora de Carrera "G" y Coordinadora de Seguimiento del Egresado ** Profesora de Carrera "G" y Coordinadora del Programa de Nutrición *** Profesora de Carrera "G" y Coordinadora de Servicio Social **** Profesora de Carrera " $\mathrm{G}$ " y Coordinadora de Tutorias ***** Profesora de Carrera " $\mathrm{G}$ " y Coordinadora de Difusión ****** Profesora de Carrera "G" y Coordinadora de Planeación

Unidad Académica Multidisciplinaria Matamoros de la U niversidad Autónoma de Tamaulipas (U AM M-U AT)

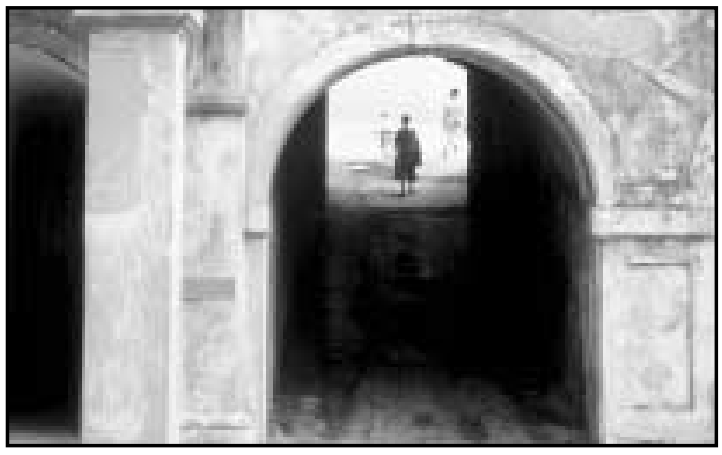

NURSING INTERVENTION WITHIN SELFCARE WITH THE EDUCATIONAL SUPPORT TO PEOPLE WITH TYPE 2 DIABETES MELLITUS

\section{SUMMARY}

Introduction: The purpose of this study was to determine how nursing interventions influence self-care of individuals with type 2 Diabetes mellitus, with educational support.

Methodology: The design was descriptive, pre-experimental, and longitudinal. The sample was probabilistic for convenience. The sample was composed of 20 individuals with type 2 diabetes mellitus. The instrument Scale of Capacity for Self-Care was used having a Cronbach Alpha of
0.7086. Two measurements were made: one prior to the nursing intervention, the second after implementing an educational support program lasting 8 hours ( 2 hours per day). Measurements were made using the "paired-sample t test" to determine the difference between pre and post -test.

Results: The average age was 50 to 59 years old, and the distribution by sex was predominantly female, $75 \%$. With respect to marital status, $75 \%$ of the individuals were married. Using a "pairedsample t test" it was observed that a significant difference exists. Pre-test and post-test results show the statistical significance of nursing interventions on the capacity of self care after receiving educational support, t3.579.p.002.

Discussion: Statistical significance was observed in the second measurement after nursing interventions were implemented and post-test conducted. This agrees with authors Gayosso, Oller and Agramone, and Otero who reported an increase in post test results.

\section{RESUMEN}

Introducción: El propósito fue conocer de qué manera influyen las intervenciones de enfermería en el autocuidado de las personas con Diabetes Mellitus tipo 2 (DMT2) con apoyo educativo. 
Metodología: El diseño fue descriptivo, preexperimental, longitudinal. El muestreo fue probabilístico por conveniencia. El tamaño de la muestra estuvo conformada por 20 personas con diabetes mellitus tipo 2. Se utilizó el instrumento cuestionario Escala de Capacidades de Autocuidado siendo un instrumento con un Alpha de Cronbach de 0.7086.Se realizaron dos mediciones: una antes de la intervención de enfermería, después se implementó un programa de apoyo educativo, con una duración total de ocho horas (dos horas diarias), al término del programa se realizó la segunda medición utilizando la t pareada para conocer la diferencia entre la preprueba y posprueba.

Resultados: El promedio de edad estuvo en el rango 50-59 años y la distribución por género predominó el sexo femenino (75\%). Con respecto al estado civil, la mayoría señaló ser casado (75\%). Utilizando la prueba t pareada se observó que existe diferencia significativa. En los resultados de preprueba y la posprueba de la intervención de enfermería se obtuvo significancía estadística en la capacidad de autocuidado después de recibir apoyo educativo de enfermería t-3.579 p.002. Discusión: En la segunda medición se observó que existe significancia estadística en las intervenciones de enfermería posprueba. Esto concuerda con los autores Gayosso, Oller \& Agramonte, Otero quienes reportaron incremento en la posprueba.

\section{Palabras clave: Autocuidado, Apoyo} Educativo, Diabetes Mellitus Tipo 2

\section{INTRODUCCIÓN}

La diabetes mellitus tipo 2 (DMT2) es una de las enfermedades crónicas degenerativas que se presenta con mayor frecuencia en la población mundial, al grado de considerarse una pandemia con tendencia ascendente. En la actualidad, y de acuerdo a la Organización Mundial de la Salud (OMS, 2004.), se estima que existe alrededor de 140 millones de personas con esta enfermedad en el mundo y se espera que esa cifra se eleve a 300 millones en los próximos 25 años (Mora, 2003). El aumento será de $40 \%$ en los países desarrollados y el $70 \%$ en los países en vías de desarrollo (Alpizar, 2001)
En México, DMT2 es un problema de salud pública, ocupa el primer lugar de mortalidad general. Para el año 2000 la prevalencia en individuos mayores de 20 años es de $7.5 \%$, lo anterior representa un total de 2.8 millones de adultos diagnosticados con este padecimiento (Encuesta Nacional de Salud [ENSA] 2000, p. 93-102 ). La prevalencia fue ligeramente mayor en mujeres que en hombres (7.8 Vs $7.2 \%$ respectivamente). Una proporción importante de casos nuevos fue en personas menores de 40 años, lo que incrementa el riesgo de complicaciones crónicas de la enfermedad a temprana edad. Un dato importantes es la relación entre la presencia de diabetes y el nivel de escolaridad ya que se da inversamente proporcional, es decir se presenta la enfermedad con mayor frecuencia en grupos de menor escolaridad (ENSA, 2000).

En Tamaulipas la DM se encuentra ocupando el $8^{\circ}$. lugar en morbilidad, y en mortalidad el $3^{\text {er }}$. lugar. (Instituto Nacional de Estadística y Geografía [INEGI], 2000, ). El efecto de las enfermedades crónicas ha sido tradicionalmente evaluado a través de la mortalidad y morbilidad; sin embargo, en los últimos años aparecen más publicaciones que abordan métodos diagnósticos, avances terapéuticos, aspectos dietéticos, pero son pocos los estudios que se refieren al impacto que tiene la intervención de enfermería a través del autocuidado por medio de la educación a pacientes con DM (García, Reyes, Garduño, Fajardo \& Martínez. 1995 p.293-298 ).

La educación para la salud es la forma más frecuente de intervención profesional de las enfermeras con el paciente diabético, pero no es la más efectiva para influir positivamente sobre su adherencia al tratamiento. Mantener un nivel de bienestar en presencia de la enfermedad requiere llevar a cabo una serie de acciones, de acuerdo a la Norma Oficial Mexicana para le Prevención, Tratamiento y Control de la Diabetes Mellitus, el plan de manejo debe incluir el establecimiento de metas de tratamiento, el manejo farmacológico, no farmacológico entre los que se incluyen un plan de alimentación adecuado, el ejercicio físico y control de peso, el automonitoreo y la vigilancia de complicaciones (Secretaria de Salubridad y Asistencia [SSA], 1994) 
Aún y cuando esta norma no describe al paciente como el principal responsable de su tratamiento, indica que la educación del paciente es un elemento indispensable para el control de la enfermedad, lo anterior refleja que el profesional de enfermería tiene un papel relevante para que el paciente se adhiera a dicho tratamiento. Sin embrago, diversos estudios en población diabética indican que hay otros factores emocionales de índole económico y social que pueden influir en el control de la enfermedad (García, et al.,1995) (Polly, 1993).

El supuesto que sustenta esta intervención es que si la persona conoce todo acerca de su enfermedad y de cómo cuidarse, es capaz de seguir las indicaciones de las clínicas para atención y control del paciente con diabetes. Con frecuencia los programas educativos cuyo contenido versa sobre la enfermedad y su tratamiento, se complementan con ejercicios grupales basados en conceptos de psicología y sociología, a fin de lograr un mejor aprendizaje (García, 1993)

El sistema de enfermería de apoyo-educativo se utiliza cuando un paciente puede satisfacer los requisitos del autocuidado pero necesita ayuda en la toma de decisiones, el control de la conducta o en la adquisición de habilidades (por ejemplo un paciente diabético controlado busca información adicional de la enfermedad sobre la dieta), en este sistema, la enfermera intenta promocionar el órgano de autocuidado (Wesley, 1997)

Por otro lado el cuidado como razón de ser de la practica de enfermería debe de ser brindado a las personas con y sin diagnostico de enfermedad. Uno de los indicadores del cuidado es el significado que el paciente tiene sobre el mismo, el analizar el significado de este, y además de ser un indicador de calidad de la atención de enfermería (Durán \& Pinto 1994, p.16-23).

Las investigaciones relacionadas con intervenciones de enfermería sobre el apoyo educativo en personas con diferentes patologías crónicas dan una visión de la importancia que representa esta actividad en cambios positivos en el control de las enfermedades crónico degenerativos. Por lo que la educación en diabetes, es hoy en día el aspecto más importante dentro del tratamiento integral de esta enfermedad. Se ha vuelto una herramienta indis- pensable para que el paciente sea capaz de mantener bajo control sus niveles de glucosa y pueda prevenir las complicaciones que la diabetes puede provocar a corto y a largo plazo. Esto se ha demostrado ha partir de diversos estudios acerca de la educación en diabetes que han comprobado que este proceso permite a los pacientes elevar su calidad de vida y evitar las complicaciones que esta enfermedad puede provocar hasta en un $80 \%$.

Por lo que se considera necesario realizar un estudio preexperimental, de series en el tiempo cuyo principio básico es la conducta de investigar durante un periodo largo y la introducción de un tratamiento experimental durante la obtención de los datos (Polit 2000 p.) para determinar la intervención de enfermería en el autocuidado con apoyo educativo en personas con DMT2. El propósito fue realizar un estudio de tipo descriptivo que permitió conocer de que manera influye significativamente las intervenciones de enfermería en el autocuidado de las personas con diabetes mellitus tipo 2 con apoyo educativo.

\section{OBJETIVO GENERAL}

Conocer como influye la intervención de enfermería en el autocuidado de las personas con diabetes Mellitus tipo 2 con apoyo educativo.

\section{Específicos}

1. Proporcionar apoyo educativo a las personas con Diabetes Mellitus Tipo 2 de la colonia las Culturas de la Cuidad de Matamoros Tamaulipas con intervenciones de enfermería

2. Medir la capacidad Autocuidado antes y después en las personas con diabetes Mellitus tipo 2 de la Colonia las Culturas de la cuidad de Matamoros Tamaulipas

\section{METODOLOGÍA}

El diseño del estudio fue descriptivo (Polit \& Hungler, 2000) pre-experimental, longitudinal La población de estudio estuvo conformada por 77 personas con diagnóstico de Diabetes Mellitus Tipo 2 de ambos sexos de la colonia las Culturas de H. Matamoros, Tamaulipas. El muestreo fue probabilístico por conveniencia ya que se acudió a los domicilios para solicitarle su participación. El tamaño de la muestra fue de 31 sujetos de estudio 
se consideró como criterio la permanencia a las intervenciones quedando solamente 20 sujetos. Criterios de inclusión, sujetos diagnosticados con DMT2 de ambos sexos, edad de 25 a 75 años. Se les solicitó su permiso mediante el consentimiento informado, se corroboró a través de preguntas sencillas de nombre, edad, fecha y lugar, que estén ubicados en lugar, tiempo y espacio así como asistencia permanente a las intervenciones de enfermería programadas

Instrumento: El instrumento que se utilizó se llama Escala de Capacidades de Autocuidado de los autores Rosalina Díaz Guerrero, Ma. Lourdes Jordán Jinez, Ana María Vera Ramírez, Laura Ruíz Paloalto, Eva Olalde García. El propósito fue conocer requisitos de autocuidado universales, de desarrollo y de desviación de la salud, así como los datos sociodemográficos. El instrumento está constituido por dos partes, la primera son los datos de identificación que incluye los factores condicionantes básicos, entendiéndose estos como condiciones que afectan los valores o las formas de cubrir los requisitos de autocuidado que afectan el desarrollo o adecuación de las capacidades de la persona para cuidar de sí misma y de los que dependen de ella.

Para el llenado en ese apartado en el espacio del nombre se anotaron las siglas del paciente para guardar su anonimato, además, edad, género, estado civil, domicilio, escolaridad y religión.

La segunda parte está constituida por 25 ítems que evalúan los requisitos de autocuidado universales, de desarrollo y desviación de la salud, se anotan delante de cada uno de ellos una $X$ en el recuadro que considere más aproximado a la frecuencia con que el paciente practica las acciones descritas en cada enunciado. Cada ítem está clasificado con valores de cero (nunca), 1 (a veces ), 2 (frecuentemente) y 3 (siempre), se hace la sumatoria de las cuatro categorías de respuesta y se compararon con la siguiente puntuación; Capacidades de Autocuidado con puntuaciones de 57 - 75 (muy buena), de $38-56$ (buena), de 19-37 (regular) por último de 00-18 (mala).

La recolección de datos: una vez obtenida la autorización de la Jurisdicción Sanitaria III de Matamoros, Tamaulipas, se acudió al Centro Comunitario ubicado en la colonia las Culturas para informar al equipo de salud el objetivo del proyecto de investigación. Posteriormente se identificó la población de estudio a través de recorridos casa por casa una vez obtenido el número de los sujetos de estudio. Se les hizo la invitación a las personas con diagnóstico de DMT2 a participar en el estudio, explicándoles el propósito del mismo y que su participación consistió en asistir a las intervenciones programadas, informándoles el número de intervenciones de enfermería y se les aplicó por dos ocasiones un cuestionario que no llevó más de 20 minutos, el cual fue anónimo y confidencial. Una vez que aceptaron, se solicitó la firma del consentimiento informado y se procedió a la primera aplicación del cuestionario y se dio inicio a la intervención de enfermería con las características que las autoras Díaz \& cols. recomiendan el programa de intervención de apoyo educativo de enfermería se llevó a cabo con un tiempo de duración de ocho horas, distribuidas en cinco sesiones, la primera sesión tuvo una duración de una hora tratándose el tema de generalidades de la DMT2, la segunda sesión consistió en los cuidados del paciente, procedimientos necesarios para su atención (dieta, ejercicio, tratamiento medicamentosos, higiene) con una duración de cuatro horas, en la tercera sesión se trató el tema de promoción de la salud y protección específica con una duración de una hora, la cuarta sesión tuvo una duración de una hora, tratándose el tema de motivación para las personas con DMT2 y por último, la quinta sesión fue impartida en una hora con el tema participación de la agencia del cuidado. Al finalizar el programa se cito a los personas para la segunda medición con un Intervalo de quince días para la segunda medición.

Análisis de resultados. Los resultados obtenidos se analizaron por el paquete estadístico Statistical Package for the social Sciences (SPSS),versión 12, se utilizó estadística descriptiva para describir características de la muestra y las variables de estudio La prueba de $t$ de Student permite analizar la diferencia entre dos poblaciones.

\section{RESULTADOS}

La muestra estuvo compuesta por 20 adultos mayores con DMT2. El promedio de edad estuvo en el rango 50-59 años, la distribución por género 


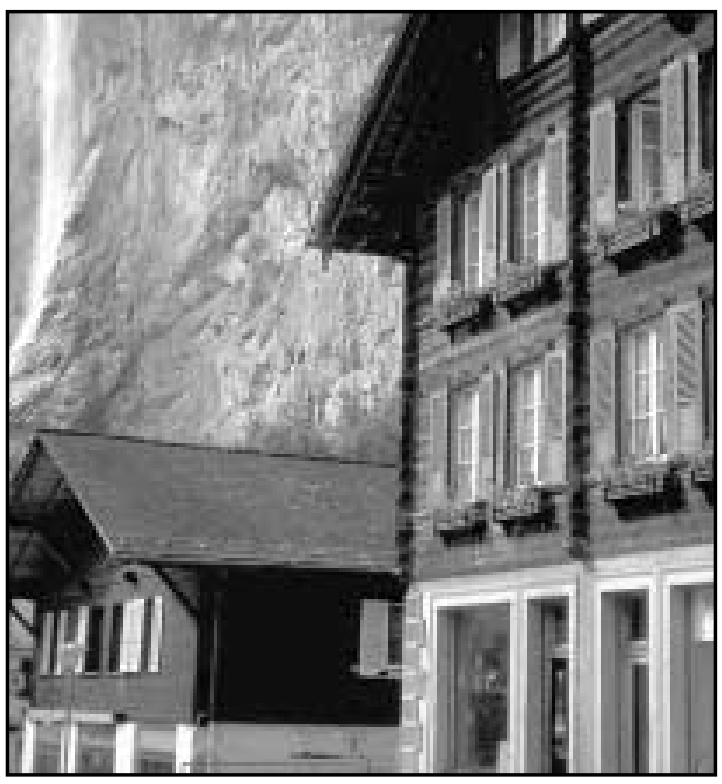

predominó el sexo femenino (75\%).Con respecto al estado civil la mayoría señaló ser casado (75\%).

Se encontró un nivel muy bajo de escolaridad en la muestra del estudio (40\%) de 0 al tercer grado de primaria, sin embargo el $35 \%$ cursó la primaria completa. La ocupación de la población se identificó que predominan las labores del hogar (75\%) lo que se debe a que en la muestra predominó el sexo femenino. Respecto a la dependencia económica se encontró que (75\%) son dependientes. El sistema de salud al que acuden los sujetos de estudio fue en un $(50 \%)$ al seguro Social y solo él $25 \%$ acude al Hospital General. Utilizando la prueba t pareada que observó que existe diferencia significativa en los resultados de preprueba sin intervención y la posprueba después de la intervención de enfermería Obteniendo significancía estadística en la capacidad de autocuidado después de recibir apoyo educativo de enfermería t-3.579 p.002.

\section{DISCUSIÓN}

El objetivo del estudio fue conocer como influye la intervención de enfermería en el autocuidado de las personas con diabetes mellitus tipo 2 con apoyo educativo antes y después de las intervenciones de enfermería se observo que existe significancía estadística en la intervención de enfermería posprueba este hallazgo concuerda con lo reportado por (Gayosso 2002, p. 292 -295) quien reporto que las personas con DMT2 mostraron significancía estadística en la pos prueba. al igual que Oller \& Agramonte (2002) quienes Obtuvieron incremento en las respuestas al cuestionario antes y después de la intervención respecto al conocimiento de las personas con DMT2 ante su autocuidado y control .Otero (2003) reporto significa en la intervención para medir el conocimiento alcanzado de la enfermedad en la población de estudio.

Las características como edad, genero, estado civil reportadas en la investigación concuerdan con lo estudiado por (Gayosso 2002), Otero (2003) Puente et al (1999)

\section{CONCLUSIONES}

Las personas con DMT2 reportaron altos índices para el autocuidado con apoyo educativo estos estuvieron relacionados con la edad, estado civil y género.

Permitieron observar cambios importantes en cuanto a la capacidad de autocuidado en los sujetos estudiados reflejando así el impacto potencial de intervención de enfermería con apoyo educativo.

\section{RECOMENDACIÓN}

Que el apoyo educativo sea permanente en los centros comunitarios de atención a la salud en forma planeada y desarrollado por profesionales de enfermería para incremento de la capacidad de autocuidado, para mejorar su estilo y calidad de vida.

En un periodo no mayor de seis meses realizar una nueva medición para fortalecer el autocuidado.

Existe interés en los autores en incrementar la muestra en futuras investigaciones en este tipo de estudio para conocer más respecto a la variable dependiente.

\section{BIBLIOGRAFÍA}

- Alpizar, S. M. (2001) Guía para el Manejo Integral del paciente Diabético. Manual Moderno, México, D.F (pp.1)

- Duran Villalobos M. (1994). El cuidado: Pilar Fundamental de Enfermería. En Ponencia presentada en el II Congreso de Actualización en Enfermería. Revista Avances en Enfermería ACOFEN. Bogotá, XII (16-23).

- García, P. M., Reyes, M. H., Garduño, E. J. Fajardo G. A. \& Martínez, G. C. (1995)- La

- Calidad de vida en los pacientes con Diabetes Mellitus tipo 2 y factores relacionados .Revista Medica IMSS 33:293-298

- García, G. R.,Suárez, P. R \& Acosta, F. O (1993) Capacidades 
de autocuidado del adulto con diabetes tipo 2.Programa de Educación en Diabetes del Instituto Nacional de Endocrinología en Cuba. Una Estrategia de Comunicación interactiva .Asociación Latinoamericana de Diabetes (11-115)

- Gayosso.,I. E.(2003) Influencia del Apoyo Educativo de Enfermería en el autocuidado del paciente con diabetes Mellitus Tipo 2.Revista de Desarrollo Científico de Enfermería . 11 (10) 292 -295

- Instituto Nacional de Estadística, Geografía e Informática [INEGI]. (2000).Estadísticas vitales del estado de Tamaulipas. Consultado el 8 noviembre de 2002, pagina web de http://www.inegi.gob.mx/

- Mora, F. J (2003) Mensaje del Secretario de Salud en la inauguración del 13 congreso Nacional Mexicano de Diabetes León Guanajuato, México. Federación Nacional de Diabetes

- Organización Mundial de la Salud.[OMS],(2004). Consultado el 20 de Junio del 2004 Actuemos contra la diabetes, ya., pagina web http://www.who.int/mediacentre/releases/2004/pr 31/es/

- Oller., G. J \& Agramonte., M. M.(2002). Programa de Educación del Diabético evaluación de su efecto. Revista Cubana Med ,18.(4) 1-9

- Otero.,L. M(2003) Impacto de un programa de promoción de la salud aplicado por enfermería a pacientes diabéticos tipo 2 en la comunidad. Revista Latino-Americana de Enfermería.11 (6)711-719

- Pinto, A. N \& Sánchez, H .V (1994) El reto de los cuidadores familiares de personas en situación crónica de enfermedad. Unilibros Bogota Colombia.

- Polly, K. R. (1993). Creencias sobre la diabetes, Conductas de autocuidado y Control glicémico entre los adultos mayores con diabetes mellitus no insulinodependientes. Public Health, 4. (21-26).

- Polit, D. F. \& Hungler. B. P. (1999). Investigación en ciencias de la salud. México :McGraw Hill Interamericana.

- Puente.,G. G Salinas.,M. A.,Villarreal.,R.E.,Garza.,E. M .,Albarran G. T \& Elizondo G. R (1999).Estrategias educativas para el control del paciente diabético tipo 2, Revista de Enfermería 7 (2) 93-98.

- Secretaria de Salud (1994). Norma Oficial Mexicana NOM015-SSA2"PARA LA PREVENCION, TRATAMIENTO Y CONTROL DE LA DIABETES MELLITUS EN LA ATENCION PRIMARIA". Consultado el 06 de Julio del 2002 en , pagina web http://www.codamedver.gob.mx/NOMs.htm

- Wesley.,L .R (2ed).(1997). Teorías y Modelos de Enfermería Springhose Notes México. McGraw-Hill Interamericana.

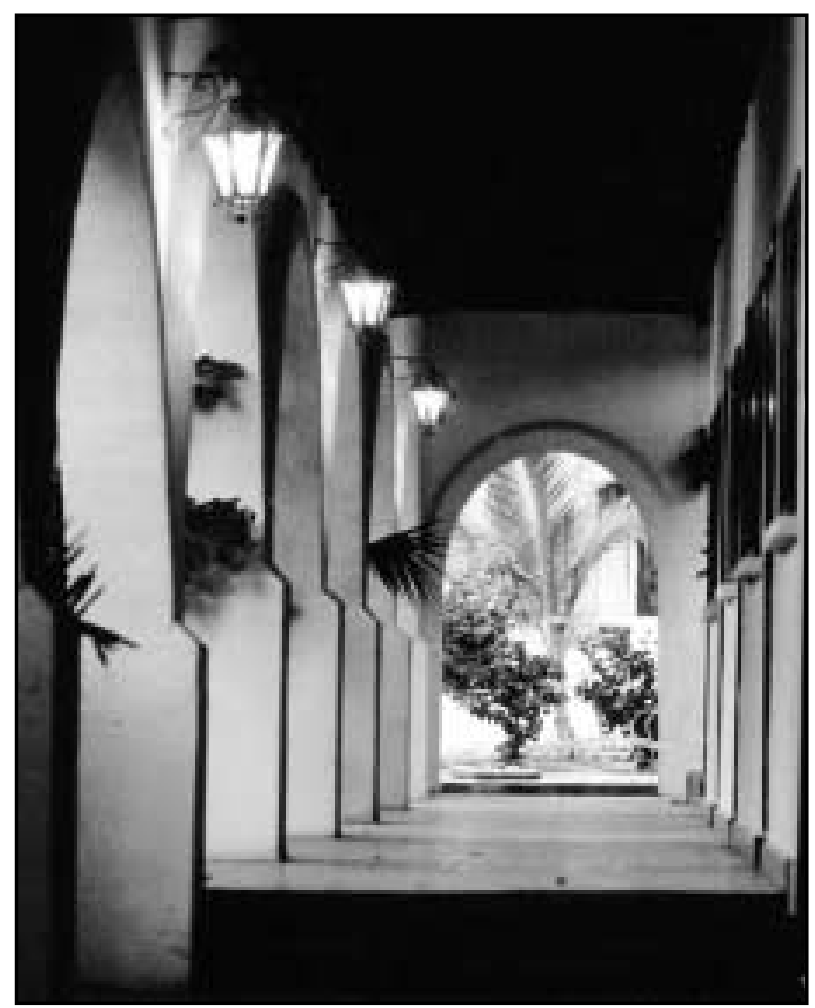

